\title{
Therapeutic and prophylactic potential of anti-microbial peptides against coronaviruses
}

\author{
Hamed Memariani ${ }^{1,2}$ (D) Mojtaba Memariani ${ }^{1,3}$ (D) \\ Received: 28 March 2020 / Accepted: 3 April 2020 / Published online: 18 April 2020 \\ (C) Royal Academy of Medicine in Ireland 2020
}

\section{Dear Editor,}

Coronaviruses constitute a large family of enveloped positive-sense RNA viruses that can cause respiratory diseases, ranging from the common cold to life-menacing pneumonia $[1,2]$. Unlike severe acute respiratory syndrome (SARS) and Middle East respiratory syndrome (MERS), a far greater proportion of world's population has been afflicted by coronavirus disease 2019 (COVID-19). As fatalities still continue to soar, researchers scramble to re-purpose existing medications to tackle the disease. A growing number of pharmaceutical companies have embarked upon clinical trials on a litany of anti-viral drugs such as lopinavir/ritonavir (HIV-1 protease inhibitor), oseltamivir (influenza virus neuraminidase inhibitor), chloroquine phosphate (a quinoline compound with anti-malarial and anti-inflammatory properties), and remdesivir (a broad-spectrum inhibitor of viral RNAdependent RNA polymerase), to mention only a few [1].

Anti-microbial peptides (AMPs), also called host-defense peptides, are pivotal effector molecules of intrinsic defense system of virtually all multi-cellular organisms, acting as endogenous antibiotics to obliterate intruding pathogens [3]. These peptides often display short chain lengths (typically $<$ 50 amino acids), positive charges, and around $\geq 30 \%$ hydrophobicity. In addition to anti-microbial activities, AMPs may elicit immunomodulatory effects [4]. Owing to their broadspectrum anti-microbial activity, robust microbicidal potency, favorable pharmacokinetic properties, and low tendency for

Mojtaba Memariani

memaryani@gmail.com

1 Skin Research Center, Shahid Beheshti University of Medical Sciences, Tehran, Iran

2 Biotechnology Research Center, Pasteur Institute of Iran, Tehran, Iran

3 Department of Pathobiology, School of Public Health, Tehran University of Medical Sciences, Tehran, Iran resistance development, AMPs seem to be ideal drug leads for treating various infections including viral diseases [5].

In an attempt to appraise anti-viral activity of mouse $\beta$ defensin-4-derived peptides, Zhao et al. [6] found that a short peptide named $\mathrm{P} 9$ lessened in vitro infectivity of MERS-CoV (strain hCoV-EMC/2012) or SARS-CoV (strain HKU398490) at non-toxic concentrations, ranging from 0.4 to $50 \mu \mathrm{g} / \mathrm{mL}$. In this context, more than $95 \%$ inhibition of viral infectivity was observed at concentrations above $25 \mu \mathrm{g} / \mathrm{mL}$. Interestingly, both liquid and powder forms of $\mathrm{P} 9$ still retained their anti-viral potency, even after 1 year of storage at $-20^{\circ} \mathrm{C}$. In terms of anti-viral modes of action, P9 penetrated into cells together with viruses through endocytosis and repressed endosomal acidification, thereby prohibiting membrane fusion and subsequent viral genome release. Western blot assay also revealed that P9 bound to S2 of MERS-CoV spike glycoprotein [6], a subunit involving in viral membrane fusion and entrance into the host cell. Thus, P9 can thwart cellular entry of MERS-CoV by direct interaction with spike glycoprotein. Another study deciphered a concentration-related inactivation of SARS-CoV pseudovirus by Mucroporin-M1, an analogue peptide of Mucroporin retrieved from the venom of scorpion Lychas mucronatus [3]. The 50\% inhibitory concentrations of Mucroporin-M1 against SARS-CoV pseudovirus and HeLa-ACE2 cell were 14.46 and $61.58 \mu \mathrm{g} / \mathrm{mL}$, respectively, leading to a selectivity index of 4.26. This finding indicated that anti-SARS-CoV activity of Mucroporin-M1 did not arise from cytotoxic effects of the peptide. Strikingly, incubation of SARS-CoV with $20 \mu \mathrm{g} / \mathrm{mL}$ of Mucroporin-M1 for $1 \mathrm{~h}$ could almost extinguish viral infectivity [3]. It is assumed that virucidal effects of Mucroporin-M1 may be due to direct interaction of the peptide with the viral envelope.

Beyond direct anti-viral effects, some AMPs are capable of regulating expression levels of several genes involved in innate and adaptive immunity toward viral antigens. In macrophage-like THP- 1 cells, $1 \mu \mathrm{g} / \mathrm{mL}$ of a recombinant protein consisting of human $\beta$-defensin (HBD) 2 and a receptorbinding domain of MERS-CoV S protein (S RBD) has been 
observed to augment in vitro expression levels of genes associated with innate immunity such as interferon-beta (IFN- $\beta$ ), IFN- $\gamma$, tumor necrosis factor-alpha (TNF- $\alpha$ ), interleukin- $1 \beta$ (IL-1 $\beta$ ), and IL-6 [4]. In another study, rhesus peptide $\theta$ defensin-1 (RTD-1) had no inhibitory activity on viral titers in vitro and in vivo [2]. However, RTD-1 mitigated pulmonary pathology, particularly necrotizing bronchiolitis, during the infection, mainly by alteration of cytokine responses to SARS-CoV in lung tissues. Based on these findings, it seems that some AMPs have the potential to alleviate severity of coronavirus diseases indirectly by reduction of pulmonary inflammation.

Regarding in vivo studies, some AMPs were able to protect mice against coronavirus-related pulmonary disease and mortality. For example, intranasal pre-treatment of BALB/c mice with two doses $(5 \mathrm{mg} / \mathrm{kg}$ ) of RTD-1 $15 \mathrm{~min}$ before SARS$\mathrm{CoV}$ infection followed by a single dose at $18 \mathrm{~h}$ postinfection led to $100 \%$ survival and little weight loss, whereas mortality rate of around $75 \%$ and obvious weight loss were observed in infected mice receiving no RTD-1 [2]. Of note, RTD-1-protected mice surviving a lethal dose of SARS-CoV showed neither signs of morbidity nor weight loss in future exposure. In the case of peptide P9, Zhao et al. [6] highlighted both preventive and therapeutic effects against SARS-CoV in $\mathrm{BALB} / \mathrm{c}$ mice at day 3 post-infection. In this respect, one dose $(100 \mu \mathrm{g} /$ mouse $)$ of intratracheal P9 for prophylaxis and 5 doses of intranasal P9 for therapy considerably decreased viral titers in lungs of mice challenged with SARS-CoV compared with SARS-CoV-infected untreated mice $(P<0.05)$.

AMPs have the potential to be exploited as vaccine adjuvants. For instance, Kim et al. [4] found that C57BL/6 mice immunized with $10 \mu \mathrm{g}$ of the HBD 2-conjugated S RBD had significantly greater S RBD-specific immunoglobulin $\mathrm{G}$ levels in comparison with those receiving $\mathrm{S}$ RBD alone $(P<0.01)$. Likewise, sera of mice immunized with HBD 2conjugated S RBD efficiently inhibited the binding of S RBD to its receptor on Huh-7 cells [4]. These observations highlight the ability of AMP-conjugated proteins to elicit high neutralizing antibody titers.

Inhalation of AMPs as therapeutics or prophylactics represents an auspicious strategy for treatment of not only COVID19 but also other coronavirus infections. We also envisage that conjugation of AMPs with aptamers or nanoparticles will offer undoubted advantages over AMPs alone. Last but not least, combination of AMPs with current anti-viral drugs may exert potent synergistic activities as well as minimizing the emergence of drug-resistant mutants. All in all, these data will pave the way for future clinical trials of AMPs for treatment of patients with viral respiratory diseases.

Authors' contributions Both authors contributed equally for writing and revision of this manuscript.

\section{Compliance with ethical standards}

Conflict of interest The authors declare that they have no conflict of interest.

Ethics approval This article does not contain any studies with human participants or animals performed by any of the authors.

Consent for publication All authors approved the publication of the manuscript.

\section{References}

1. Fisher D, Heymann D (2020) Q\&A: the novel coronavirus outbreak causing COVID-19. BMC Med 18(1):57. https://doi.org/10.1186/ s12916-020-01533-w

2. Wohlford-Lenane CL, Meyerholz DK, Perlman S, Zhou H, Tran D, Selsted ME, McCray PB Jr (2009) Rhesus theta-defensin prevents death in a mouse model of severe acute respiratory syndrome coronavirus pulmonary disease. J Virol 83(21):11385-11390. https://doi. org/10.1128/JVI.01363-09

3. Li Q, Zhao Z, Zhou D, Chen Y, Hong W, Cao L, Yang J, Zhang Y, Shi W, Cao Z, Wu Y, Yan H, Li W (2011) Virucidal activity of a scorpion venom peptide variant mucroporin-M1 against measles, SARS-CoV and influenza H5N1 viruses. Peptides 32(7):15181525. https://doi.org/10.1016/j.peptides.2011.05.015

4. Kim J, Yang YL, Jang SH, Jang YS (2018) Human $\beta$-defensin 2 plays a regulatory role in innate antiviral immunity and is capable of potentiating the induction of antigen-specific immunity. Virol J 15(1):124. https://doi.org/10.1186/s12985-018-1035-2

5. Memariani H, Memariani M, Moravvej H, Shahidi-Dadras M (2020) Melittin: a venom-derived peptide with promising anti-viral properties. Eur J Clin Microbiol Infect Dis 39(1):5-17. https://doi.org/10. 1007/s10096-019-03674-0

6. Zhao H, Zhou J, Zhang K, Chu H, Liu D, Poon VK, Chan CC, Leung HC, Fai N, Lin YP, Zhang AJ, Jin DY, Yuen KY, Zheng BJ (2016) A novel peptide with potent and broad-spectrum antiviral activities against multiple respiratory viruses. Sci Rep 6:22008. https://doi. org/10.1038/srep22008

Publisher's note Springer Nature remains neutral with regard to jurisdictional claims in published maps and institutional affiliations. 